\title{
Conference Paper \\ Dynamic Assessment of Fibrinogen Adsorption and Secondary Structure Perturbation
}

\author{
Gaurang Khot, ${ }^{1}$ Folashade Kuforiji, ${ }^{2}$ Rupert Wright, ${ }^{2}$ and Paul Roach ${ }^{2}$ \\ ${ }^{1}$ Faculty of Bionics, Hochschule Rhine-Waal University of Applied Sciences, Marie-Curie-Straße 1, 47533 Kleve, Germany \\ ${ }^{2}$ The Guy Hilton Research Centre, Keele University, Staffordshire ST4 7QB, UK \\ Correspondence should be addressed to Paul Roach; p.roach@keele.ac.uk
}

Received 22 November 2013; Accepted 12 February 2014; Published 7 April 2014

Academic Editors: J. Gough and R. Sammons

This Conference Paper is based on a presentation given by Gaurang Khot at "UK Society for Biomaterials Annual Conference 2013" held from 24 June 2013 to 25 June 2013 in Birmingham, United Kingdom.

Copyright (C) 2014 Gaurang Khot et al. This is an open access article distributed under the Creative Commons Attribution License, which permits unrestricted use, distribution, and reproduction in any medium, provided the original work is properly cited.

Fibrinogen is a protein being of prime importance for the initiation of clotting and thrombus formation, readily adsorbed onto surfaces presenting both hydrophilic and hydrophobic nature. The mechanism of adsorption, and thus the final presentation of this protein are therefore important for subsequent involvement for, for example, platelet adhesion. Biological activity can be controlled through careful consideration of material design; here we report kinetic assessment of fibrinogen adsorption onto plasma polymerised allylamine (hydrophilic) and hexane (hydrophobic) surfaces, using FTIR-ATR to inform on kinetics of adsorption, secondary structure evaluation, and orientational variation. Fibrinogen was found to respond differently to these two surfaces, adsorbing more rapidly to hydrophilic surfaces and losing an ordered secondary structure over a much longer timescale compared to hydrophobic surfaces.

\section{Introduction}

The interface between biological fluids and the surface of a material is of the utmost importance when considering the longevity and function of materials in such environments. Biological activity at this interface is dictated by the ability of the surface to support the adsorption of proteinatious materials. Adsorption of biological species (e.g., proteins/peptides) occurs rapidly upon interaction of the biological fluid with numerous surface cues acing in synergy, such as chemical functionality and nano-scale features, to control adsorption characteristics and activity of bound species. Surface engineering therefore plays a major part in the advancement of material function; the aim within the biological sciences is the production of designer materials that specifically control biological responses. Such materials have far-reaching impact, from the success of implantable or point-of-care biotechnology, designer materials for the reduction of hospital acquired infections, to advanced cell culture materials for optimised stem cell expansion and guided differentiation. The design of advanced materials towards achieving these goals is therefore of high commercial impact and of considerable economic and social value.

Protein molecules rapidly accumulate on surfaces, starting milliseconds after contact between the solid and biofluid. This is a highly dynamic process wherein protein molecules bind, rearrange, and detach. It is well accepted that surface parameters presented by materials dictate how rapidly and to what extent specific proteins adsorb; each parameter, such as chemical functionality or nano-structure, acts synergistically giving rise to a vast amount of batch processing required to fully understand even the simplest adsorption process from single protein containing systems [1]. The mechanism of interaction and the degree of influence the surface has on the adsorbed protein structure can be elucidated by following the adsorption process.

Surface chemistry dictates the initial interaction of species diffusing to the material surface. Pockets of localised charge (and/or polarisable group distribution) on the surface of protein molecules may drive adsorption, balancing electrostatic charge and interaction of hydrophobic moieties. 
The concentration of protein solution from which adsorption is occurring has also been shown to have an impact on the packing orientation/conformation, likely due to intermolecular interaction during adsorption [2]. Depending on the strength of inner bonding holding the peptide secondary structure in conformation, and the overall protein-material affinity, the adsorbing molecule may deform (denature) to maximise binding efficiency. Therefore, it may be expected that protein structural change occurring during the adsorption process may dictate the presentation of the equilibrated protein layer. Adsorption therefore influences biological function, impacting the ability to interact/communicate with cell membrane receptors [3].

Fibrinogen is a highly abundant plasma glycoprotein, being rod-shaped with a highly flexible $\sim 40 \mathrm{~nm}$ long axis. Primarily involved in the coagulation cascade, fibrinogen levels increase during inflammation, with high levels known to increase risk of coronary artery disease [4] and stroke [5]. Clearly materials design must be considering for those in contact with fibrinogen containing biofluids, particularly where thrombus formation could result in major adverse pathology. Surface coatings of plasma polymerised allylamine and hexanes are well used within the biomaterials community as model surfaces with which to present biologically relevant amine hydrophilic containing and hydrophobic hydrocarbon surfaces, respectively [6]. Here we report the kinetic investigation of fibrinogen onto these surfaces, taking into account the structural/orientational changes occurring during this process.

\section{Experimental}

2.1. Materials. Fibrinogen was purchased as a lyophilised power (Enzyme Research, Swansea) and re-suspended in fresh PBS (Sigma, $200 \mathrm{mmol}, \mathrm{pH} \mathrm{7.4)}$ ) to a final concentration of $1.0 \mathrm{mg} / \mathrm{mL}$ immediately prior to use. Solutions were vortex stirred to ensure complete dissolution. Allylamine and hexane were purchased from Sigma and Fisher Scientific respectively, and used without further purification.

\subsection{Methods}

2.2.1. Surface Modification. The surface of a 12-bounce zinc selenide attenuated total reflectance (ATR) crystal was briefly cleaned with acetone and isopropanol, air dried, and immediately placed in a Diener Electronic Femto plasma reactor. Allylamine was degassed immediately prior to use and mounted onto the monomer inlet port. Surface treatment was carried out at $0.1 \mathrm{mbar}$, igniting plasma at $20 \mathrm{~W}$ for 5 minutes. Surface characterisation was carried out using drop shape analysis of $\mathrm{dH}_{2} \mathrm{O}$, dispensing $5 \mu \mathrm{L}$ drops onto the surface and capturing an image directly into Image J. FTIR was also carried out using the pre and posttreated ATR crystals as background and sample respectively.

2.2.2. Study of Kinetics. Protein adsorption was followed on a Thermo Scientific IS50 FTIR spectrometer fitted with a 12-bounce ZnSe ATR. A background of PBS was taken immediately prior to addition of the protein solution, with an average of 64 scans being taken at $4 \mathrm{~cm}^{-1}$ resolution. Omnic Macros Basic was used to automatically collect sequential spectra every minute over $\sim 8$ hours. Data was collected with analysis carried out in Omnic v9 and Excel. Amide I band peak fitting was carried out using Peak Resolve within Omnic, using previously reported values for component bands [2].

\section{Results and Discussion}

3.1. Protein Accumulation on the Materials Surface during Adsorption. A kinetic assessment of protein adsorption was carried out using FTIR-ATR. Over time the increase in absorbance bands indicative of protein was observed, with particular focus on the amide I and II bands, Figure 1. The sequential spectra clearly show an increase in absorbance of all the major protein peaks, demonstrating the utility of this method for kinetic assessment of relative protein quantification during adsorption, whilst also collecting information on protein structure.

An initial rapid increase was observed, progressively slowing over time. This trend has been observed by others using amide I and II as an indicator for relative adsorption of fibrinogen [7].

Here we present data with respect to amide peak areas, Figure 2. Little difference in noise levels was observed between amide I and II bands, on either plasma polymerised allylamine and hexane surfaces, used here as model hydrophilic and hydrophobic surfaces respectively. Fibrinogen adsorbed rapidly to both surfaces is an indication of a good interaction with regards to both the positively charged amine groups, whilst also indicating a hydrophobic interaction with the hexane surface.

\subsection{Change in Secondary Structure and Orientation during} Adsorption. The amide I band is highly indicative of protein secondary structure, being a compound band of many component spectral carbonyl stretches. During adsorption the amide I bands of fibrinogen were found to change shape globally, with particularly visible differences at $\sim 1680$ and $1645 \mathrm{~cm}^{-1}$. These changes can be seen in Figure 1, with an apparent decrease in FWHM over time. Component peak fitting of the amide I band highlights the variance in secondary structure over time. A gradual, but continual decrease in alpha-helical and beta-sheet structure and increase in more disordered beta-turn and open chain network was observed when fibrinogen adsorbed onto poly (allylamine), Figure 3. In contrast, variation in secondary structure on plasma polymerised hexane was not observed after initial adsorption for 2 minutes. The formation of beta-sheet structure can be rationalised by the proportion of proline and glycine residues within fibrinogen, which are prone to forming betasheets [8].

The ratio of amide $\mathrm{I} / \mathrm{II}$ is also indicative of protein structural/orientational change. Although spectra displayed relatively unchanged ratio for fibrinogen adsorbing onto the allylamine surface, an initial decrease was observed during adsorption onto plasma polymerised hexane up to $\sim 100$ 


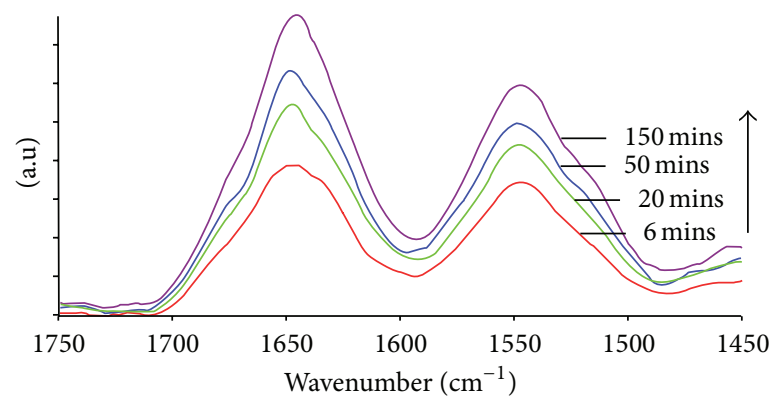

FIGURE 1: Fibrinogen adsorption followed onto a plasma-deposited hexane polymer.

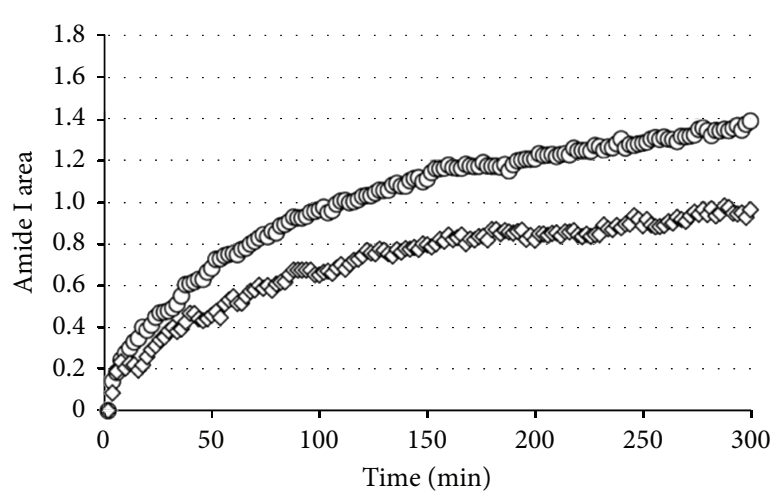

- Amide I allylamine

$\diamond$ Amide I hexane

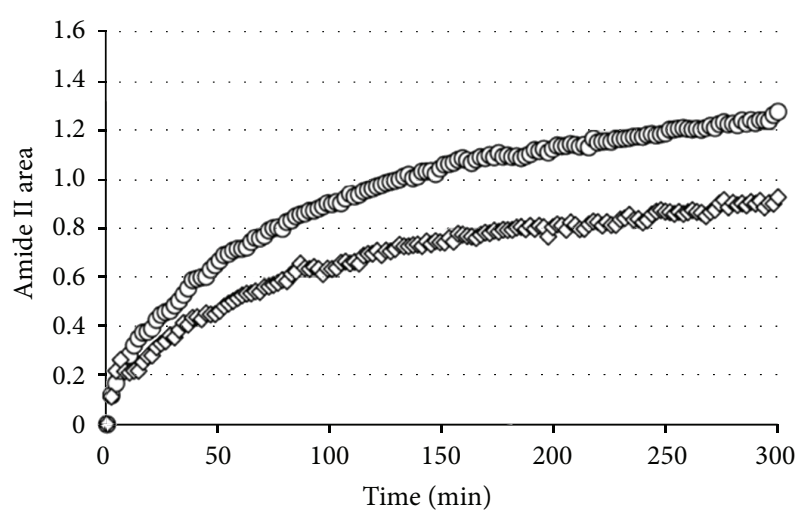

○ Amide II allylamine

$\diamond$ Amide II hexane

(a)

(b)

FIGURE 2: Kinetic assessment of amide I and II bands during fibrinogen adsorption onto plasma polymerised allylamine and hexane surfaces.

minutes, Figure 4. These data suggest progressive secondary structural changes in fibrinogen adsorbing to allylamine surfaces, with minor orientational change over the time studied here. Very rapid changes could possibly be occurring at the early stages as previously reported [2], but are not observed here due to time scale of data acquisition. On plasma polymerised hexane surfaces, a rapid dislocation of fibrinogen secondary structure elements is observed during initial stages of adsorption, with an extended period of orientational relaxation.

\section{Summary}

Fibrinogen was found to adsorb rapidly onto both surfaces tested, although at a faster rate and in greater amounts onto plasma polymerised allylamine compared to hexane. Both amide I and II adsorption bands gave rise to similar adsorption kinetic curves, being good indicators of protein accumulation at surfaces. Although adsorption was rapid, changes in secondary structure were observed well after initial adsorption. Secondary structure changes were observed on allylamine-coated surfaces, whilst orientational variation was more pronounced on hexane surfaces. On hydrophilic allylamine surfaces a gradual decrease in ordered secondary structure is observed over the time of the experiments, suggesting that conformational equilibrium was not reached within this time. Fibrinogen adsorbing onto hydrophobic hexane surfaces, possibly having a stronger overall affinity for this surface due to hydrophobic interactions, become denatured very rapidly upon adsorption, with their ordered secondary structure steadily decreasing over time as relaxation occurs until the protein layer reaches equilibrium.

\section{Conclusions}

Changes in fibrinogen adsorption mechanisms are important when considering surface engineering aspects, in relation to the control or fibrinogen activity. The presentation of this molecular layer is essential to thrombus formation adjacent to materials, with both secondary structure and oriental characteristics likely dominating the biological activity at the surface. Further work is necessary to understand how each of these impacts fibrinogen's ability to effect coagulation and steer platelet adhesion. Such findings will continue to inform the development and assessment of biomaterials. 


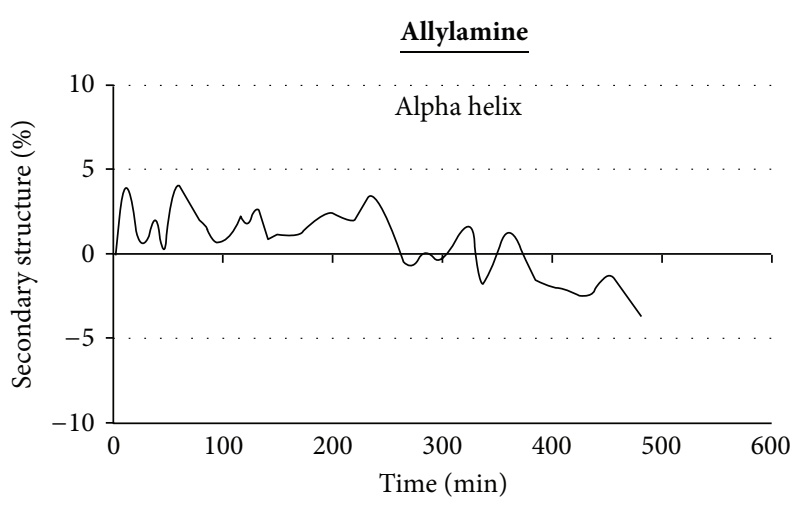

(a)

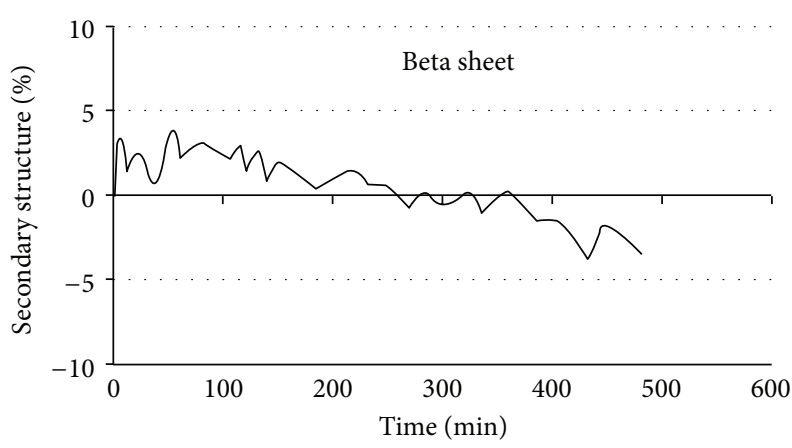

(c)

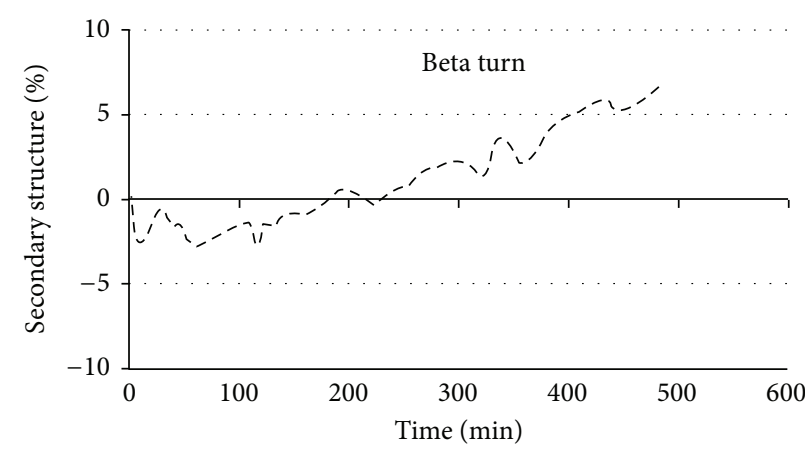

(e)

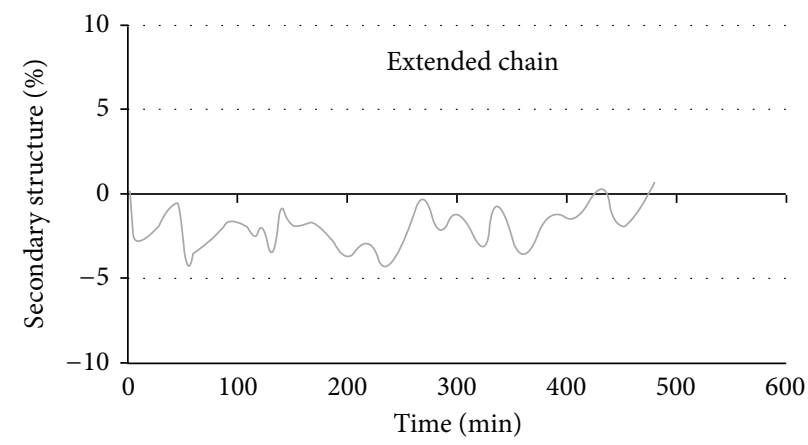

(g)

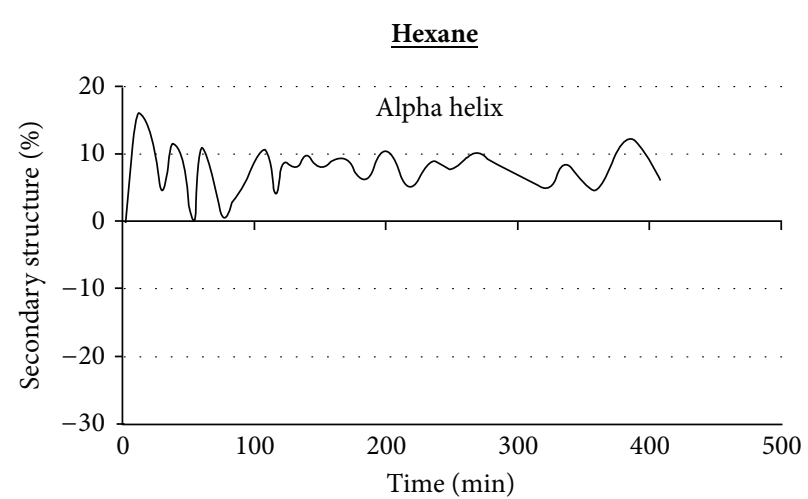

(b)

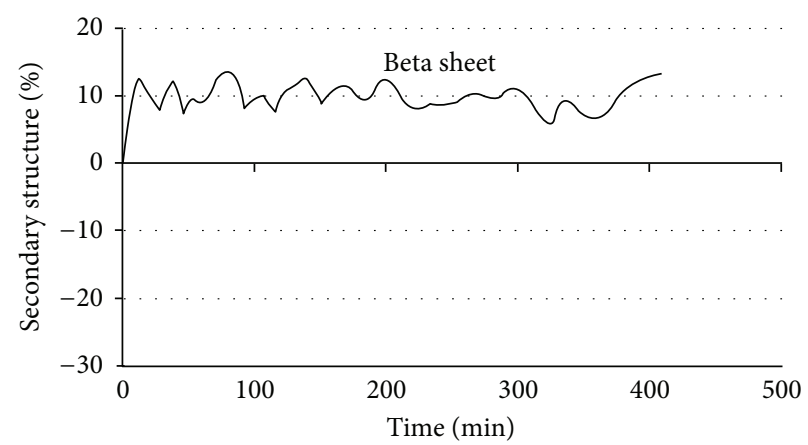

(d)

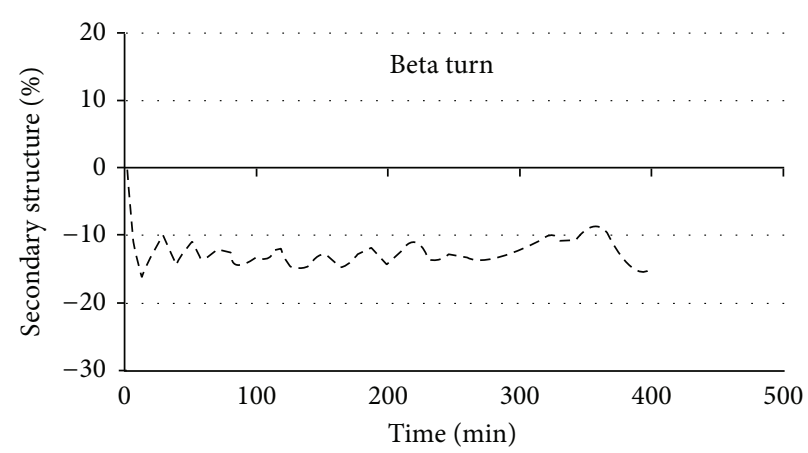

(f)

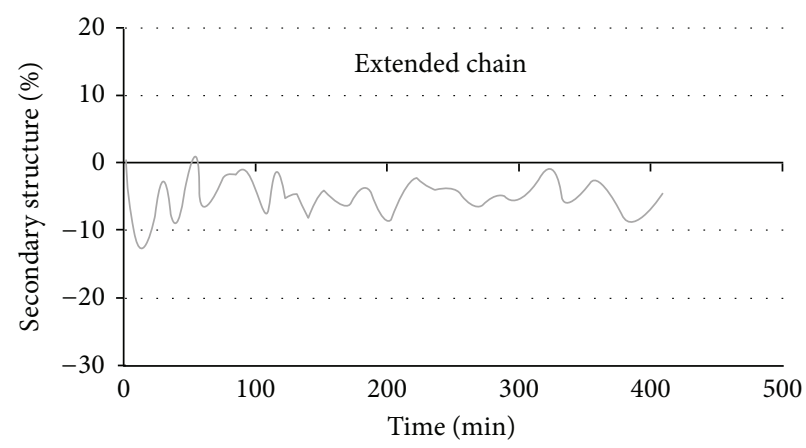

(h)

FIGURE 3: Variation in secondary structure of fibrinogen adsorbing onto plasma polymerised allylamine and hexane surfaces. 


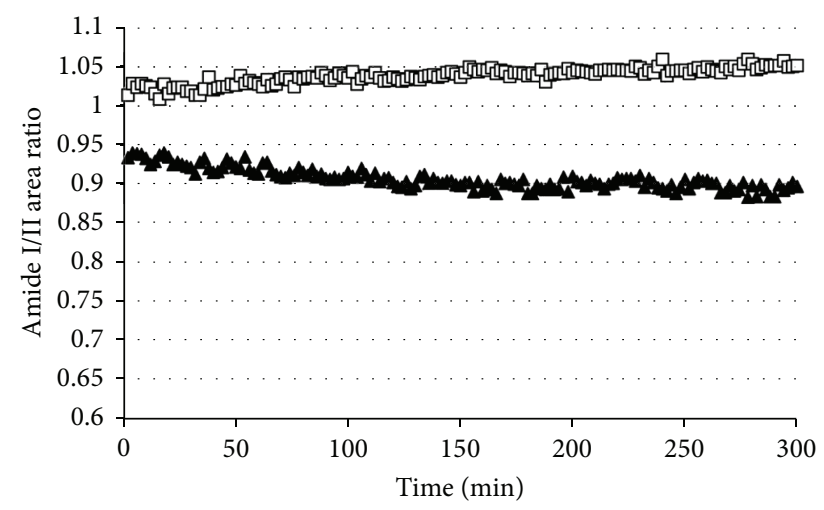

․ Allylamine

^ Hexane

FIGURE 4: Area ratios of amide I and II adsorption bands for fibrinogen adsorbing onto plasma polymerised allylamine and hexane.

\section{Conflict of Interests}

The authors declare that there is no conflict of interests regarding the publication of this paper.

\section{Acknowledgments}

We acknowledge funding support from the ERASMUS student exchange programme.

\section{References}

[1] P. Roach, D. Eglin, K. Rohde, and C. C. Perry, "Modern biomaterials: a review-Bulk properties and implications of surface modifications," Journal of Materials Science: Materials in Medicine, vol. 18, no. 7, pp. 1263-1277, 2007.

[2] P. Roach, D. Farrar, and C. C. Perry, "Interpretation of protein adsorption: surface-induced conformational changes," Journal of the American Chemical Society, vol. 127, no. 22, pp. 8168-8173, 2005.

[3] C. González-García, D. Moratal, R. O. C. Oreffo, M. J. Dalby, and M. Salmerón-Sánchez, "Surface mobility regulates skeletal stem cell differentiation," Integrative Biology, vol. 4, no. 5, pp. 531-539, 2012.

[4] G. Ndrepepa, S. Braun, L. King et al., "Relation of fibrinogen level with cardiovascular events in patients with coronary artery disease," American Journal of Cardiology, vol. 111, no. 6, pp. 804810, 2013.

[5] G. J. Hankey, N. E. Anderson, R. D. Ting et al., "Rates and predictors of risk of stroke and its subtypes in diabetes: a prospective observational study," Journal of Neurology Neurosurgery and Psychiatry, vol. 87, no. 3, pp. 281-287, 2013.

[6] M. Zelzer, R. Majani, J. W. Bradley, F. R. A. J. Rose, M. C. Davies, and M. R. Alexander, "Investigation of cell-surface interactions using chemical gradients formed from plasma polymers," Biomaterials, vol. 29, no. 2, pp. 172-184, 2008.

[7] M. Desroches and S. Omanovic, "Adsorption of fibrinogen on a biomedical-grade stainless steel 316LVM surface: a PMIRRAS study of the adsorption thermodynamics, kinetics and secondary structure changes," Physical Chemistry Chemical Physics, vol. 10, no. 18, pp. 2502-2512, 2008.

[8] L. M. Pandey, S. K. Pattanayek, and D. Delabouglise, "Properties of adsorbed bovine serum albumin and fibrinogen on selfassembled monolayers," The Journal of Physical Chemistry C, vol. 117, no. 12, pp. 6151-6160, 2013. 

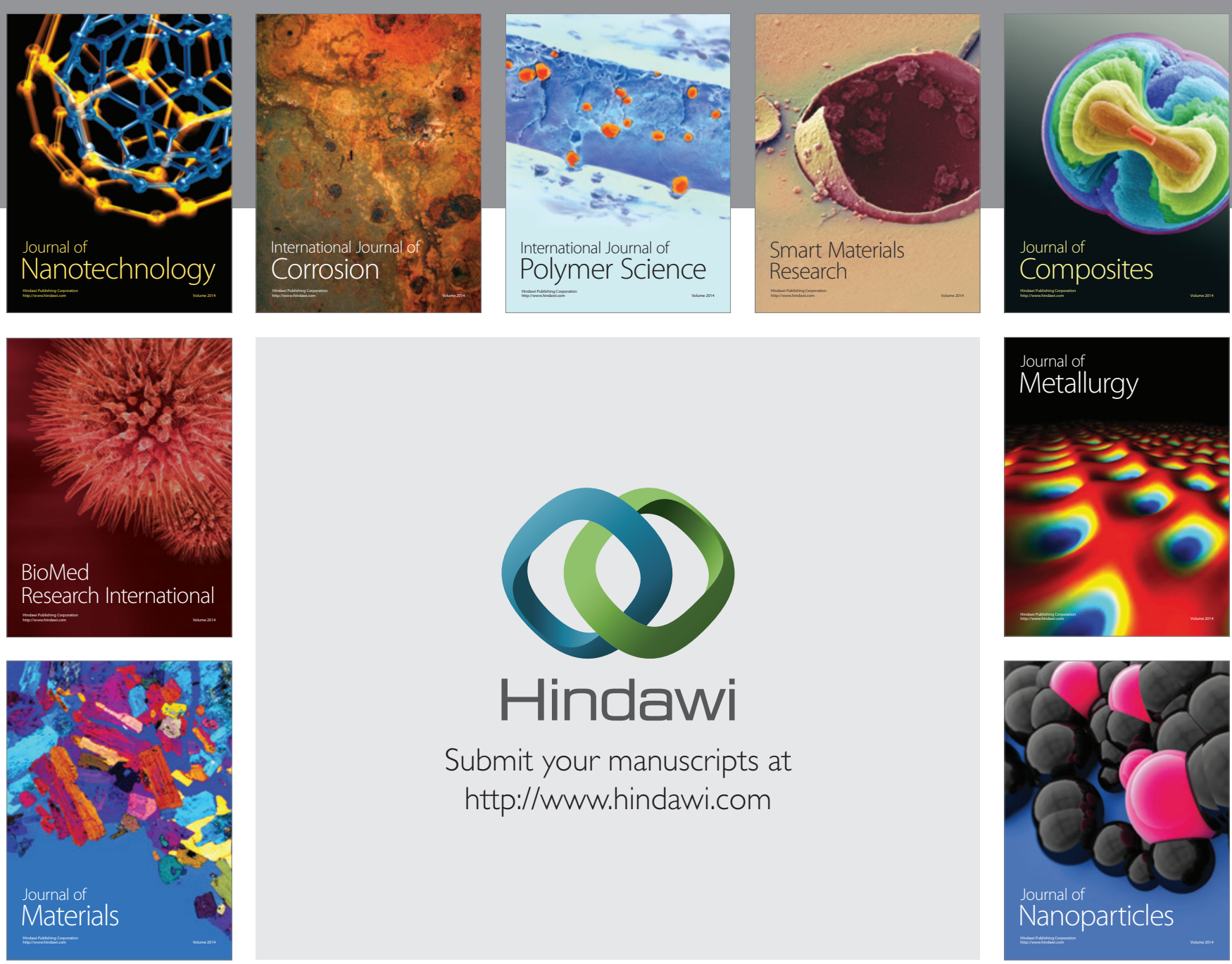

Submit your manuscripts at http://www.hindawi.com
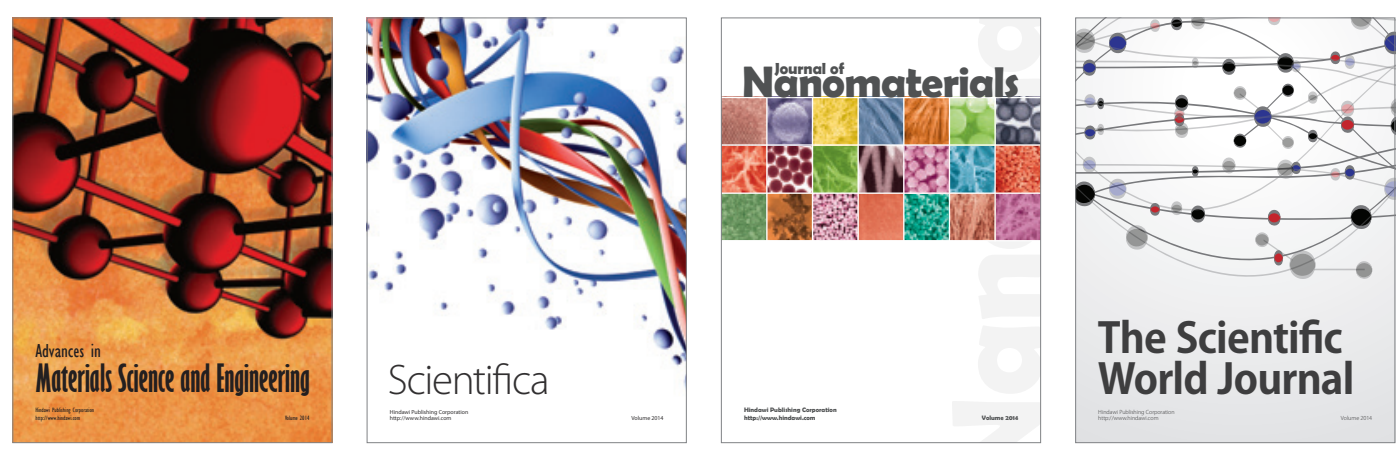

\section{The Scientific World Journal}
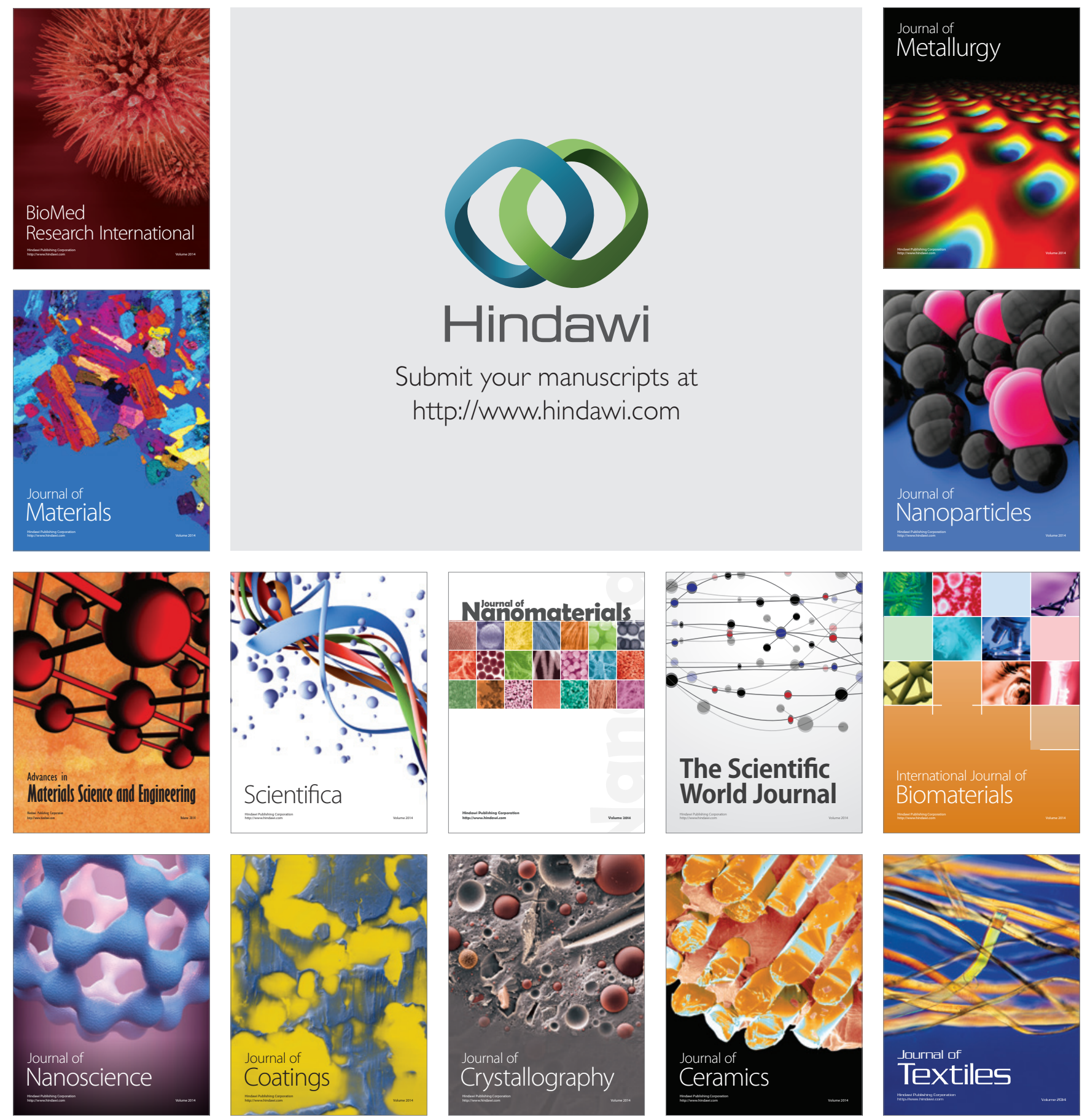